OPEN ACCESS

Edited by:

Kuangyu Shi,

University of Bern, Switzerland

Reviewed by:

Laura Pasetto,

Istituto di Ricerche Farmacologiche

Mario Negri, Italy

Stephanie Schindler,

University Hospital Leipzig, Germany

${ }^{*}$ Correspondence:

Lin Shi

shilin@cuhk.edu.hk

Dongsheng Fan

dsfan2010@aliyun.com

Received: 25 September 2020

Accepted: 16 November 2021

Published: 03 January 2022

Citation:

Ye S, Luo Y, Jin P, Wang Y,

Zhang N, Zhang G, Chen L, Shi L and

Fan D (2022) MRI Volumetric Analysis of the Thalamus and Hypothalamus

in Amyotrophic Lateral Sclerosis.

Front. Aging Neurosci. 13:610332.

doi: 10.3389/fnagi.2021.610332

\section{MRI Volumetric Analysis of the Thalamus and Hypothalamus in Amyotrophic Lateral Sclerosis}

\author{
Shan Ye ${ }^{1,2}$, Yishan Luo ${ }^{3,4}$, Pingping Jin'1,2, Yajun Wang ${ }^{1,2}$, Nan Zhang ${ }^{1,2}$, Gan Zhang ${ }^{1,2}$, \\ Lu Chen ${ }^{1,2}$, Lin Shi ${ }^{3,4 *}$ and Dongsheng Fan ${ }^{1,2 *}$ \\ 1 Department of Neurology, Peking University Third Hospital, Beijing, China, ${ }^{2}$ Beijing Municipal Key Laboratory of Biomarker \\ and Translational Research in Neurodegenerative Diseases, Beijing, China, ${ }^{3}$ Brain Research Institute, Shenzhen, China, \\ ${ }^{4}$ Department of Imaging and Interventional Radiology, The Chinese University of Hong Kong, Hong Kong, \\ Hong Kong SAR, China
}

Background: Increasing evidence has shown that amyotrophic lateral sclerosis (ALS) can result in abnormal energy metabolism and sleep disorders, even before motor dysfunction. Although the hypothalamus and thalamus are important structures in these processes, few ALS studies have reported abnormal MRI structural findings in the hypothalamus and thalamus.

Purpose: We aimed to investigate volumetric changes in the thalamus and hypothalamus by using the automatic brain structure volumetry tool AccuBrain ${ }^{\circledast}$.

Methods: 3D T1-weighted magnetization-prepared gradient echo imaging (MPRAGE) scans were acquired from 16 patients with ALS with normal cognitive scores and 16 age-, sex- and education-matched healthy controls. Brain tissue and structure volumes were automatically calculated using AccuBrain ${ }^{\circledR}$.

Results: There were no significant differences in bilateral thalamic $(F=1.31, p=0.287)$ or hypothalamic volumes ( $F=1.65, p=0.213$ ) between the ALS and control groups by multivariate analysis of covariance (MANCOVA). Left and right hypothalamic volumes were correlated with whole-brain volume in patients with ALS $(t=3.19, p=0.036$; $t=3.03, p=0.044$ ), while the correlation between age and bilateral thalamic volumes tended to be significant after Bonferroni correction $(t=2.76, p=0.068 ; t=2.83$, $p=0.06)$. In the control group, left and right thalamic volumes were correlated with whole-brain volume ( $t=4.26, p=0.004 ; t=4.52, p=0.004)$.

Conclusion: Thalamic and hypothalamic volumes did not show differences between patients with normal frontotemporal function ALS and healthy controls, but further studies are still needed.

Keywords: amyotrophic lateral sclerosis, thalamus, hypothalamus, volumetric analysis, MRI

\section{BACKGROUND}

Amyotrophic lateral sclerosis is a multiple-system neurodegenerative disease that has a far greater impact than motor system dysfunction. In recent years, more researchers in neurosciences have expanded their interest beyond motor dysfunction and explored other research axes, such as energy homeostasis and sleep disorder, in patients with amyotrophic lateral sclerosis (ALS). Patients with 
ALS generally have normal or low body mass index (BMI). Weight loss may begin before the onset of motor symptoms, which is probably caused by dysphagia and hypermetabolism. Early weight loss is associated with a higher rate of ALS progression with a grimmer prognosis (Dupuis et al., 2011; Gallo et al., 2013; O'Reilly et al., 2013; Huisman et al., 2015; Ahmed et al., 2016). Clinical trials have demonstrated that strengthening intake may benefit patients with ALS (Wills et al., 2014; Dorst et al., 2015). In addition to energy homeostasis, sleep disorders are also fairly common in patients with ALS (DiazAbad et al., 2018; Liu et al., 2018; Congiu et al., 2019). Animal studies have shown that sleep disorders may initiate before the onset of motor symptoms (Liu et al., 2015; Zhang et al., 2018). Interestingly, as both energy metabolism and sleep are closely related to the function of the hypothalamus (Vercruysse et al., 2018), it is not surprising that pathological abnormalities in the hypothalamus were found in patients with ALS (Cykowski et al., 2014; Nakamura et al., 2015). In 33 autopsy-confirmed patients with ALS, six patients presented TDP-43 pathology in the hypothalamus (Cykowski et al., 2014). In the autopsy of a patient with familiar ALS, SOD1 immunohistochemistry showed neuronal cytoplasmic inclusions, glial cytoplasmic inclusions, and dystrophic neurites in the brain and spinal cord, with a predilection for the hypothalamus and central gray matter (Nakamura et al., 2015). Hypothalamic neuropeptide levels were shown to be changed in ALS mouse models (Vercruysse et al., 2016). In addition to these biomolecular abnormalities, structural changes have also been reported: Up to $15 \%$ of patients diagnosed with behavioral variant frontotemporal dementia (bvFTD), a similar but different disease within the spectrum of ALS (Strong et al., 2017; van Es et al., 2017), were reported to have hypothalamic atrophy (Bocchetta et al., 2015; Ahmed et al., 2017). However, few studies have reported hypothalamic atrophy in patients with ALS (Gorges et al., 2017).

C9orf72 repeat expansion is one of the most important gene mutations in the ALS-FTD disease spectrum (Gijselinck et al., 2012; Balendra and Isaacs, 2018). In addition to commonly reported brain areas, such as the motor cortex, frontal and temporal lobes, and corpus callosum (Foerster et al., 2013), changes in the thalamus have been the focus of relatively few studies. Machts reported bilateral thalamic atrophy in patients with ALS-FTD (Machts et al., 2015). Schönecker's study subsequently showed thalamic atrophy in ALS/FTD C9orf72 mutation carriers that extended beyond the expected atrophy in the prefrontal and temporal subregions (Schönecker et al., 2018). However, Westeneng found that, although patients with ALS with C9orf72 repeat expansion had more severe bilateral thalamic atrophy, $21 \%$ of patients with ALS without the C9orf72 mutation had a similar neuroimaging phenotype (Westeneng et al., 2016). Chipika also found thalamic atrophy in patients with ALS without the C9orf72 mutation (Chipika et al., 2020). Some earlier studies found structural or functional changes in the thalamus in patients with ALS but lacked cognitive and behavioral assessments (Douaud et al., 2011; Sharma et al., 2011, 2013). Therefore, it remains unclear whether thalamic atrophy could occur in patients with pure ALS.

AccuBrain $^{\circledR}$ (BrainNow Ltd., Shenzhen, China) is a cloudbased National Medical Products Administration (NMPA) and Conformité Européenne (CE)-marked software tool that performs brain MRI segmentation. It is based on a multi-atlas segmentation method with a statistical anatomical atlas previously generated by experienced experts, and it automatically calculates the brain volume of various brain structures. AccuBrain ${ }^{\circledR}$ presents less inter-scanner variability than FreeSurfer and FSL-FIRST based on the comparison of their coefficient of variation values of brain volumetry, especially in basal ganglia structures, such as the thalamus $(p<0.001)$ (Liu et al., 2020). Abrigo's study showed that AccuBrain ${ }^{\circledR}$ could provide accurate automated hippocampal segmentation in accordance with the EADCADNI standard (Abrigo et al., 2018). The clinical value of AccuBrain ${ }^{\circledR}$ has also been demonstrated in various neurological disease studies (Guo et al., 2019; Wang et al., 2019; Zhao et al., 2019; Dou et al., 2020; Hou et al., 2020; Liu et al., 2020). AccuBrain ${ }^{\circledR}$ may, therefore, be a rapid and sensitive tool for detecting brain structures in neurodegenerative diseases.

The aim of this study was to determine whether thalamic and hypothalamic atrophy occurs in patients with ALS by using the AccuBrain ${ }^{\circledR}$ technique.

\section{MATERIALS AND METHODS}

\section{Participants}

In total, 16 right-handed patients who met the ALS-revised El Escorial criteria (Brook et al., 2000) at Peking University Third Hospital (PUTH) between 2011 and 2012 and 16 right-handed healthy controls were enrolled. All the patients underwent the Mini-Mental State Examination (MMSE), Frontal Assessment Battery (FAB), verbal fluency (VF), and prospective memory (PM) tests before inclusion. The exclusion criteria were as follows: (1) dementia or cognitive dysfunction, brain trauma, epilepsy, stroke, psychiatric disorders, and other central nervous system diseases; (2) absolute or relative contraindication for MRI; and (3) pregnancy.

The clinical characteristics are shown in Table 1. The patients with ALS were followed up until death; during this follow-up period, contact with eight of 16 patients was lost before this endpoint. The life span was the time from the disease onset to death. All the participants provided informed consent. The study was approved by the ethics committee of PUTH.

\section{MRI Acquisition}

The data for 16 patients with ALS and 16 healthy controls were acquired on a Siemens 3.0 T Trio TIM MR scanner. Structural MRI data were obtained using a high-resolution 3-D T1-weighted magnetization-prepared gradient echo imaging (MPRAGE) sequence [192 sagittal slices, no gap, layer thickness $=1 \mathrm{~mm}$; field of view $(\mathrm{FOV})=256 \mathrm{~mm} \times 256 \mathrm{~mm}$; repetition time $(\mathrm{TR})=2,530 \mathrm{~ms}$; echo time $(\mathrm{TE})=3.44 \mathrm{~ms}$; inversion time $(\mathrm{TI})=1,100 \mathrm{~ms}$; acquisition time $=363 \mathrm{~s}$.

\section{Brain Volumetry}

All images were processed using AccuBrain ${ }^{\circledR}$ (BrainNow Medical Technology Ltd., China), which is a brain quantification tool that performs brain structure and tissue segmentation and 
TABLE 1 | Characteristics of the participants: patients with ALS and healthy controls.

\begin{tabular}{|c|c|c|c|c|}
\hline \multicolumn{2}{|l|}{ Characteristic } & ALS patients & $\begin{array}{l}\text { Healthy } \\
\text { controls }\end{array}$ & $p$-value \\
\hline \multicolumn{2}{|c|}{ Age (years) (mean $\pm \mathrm{SD}$ ) } & $51.25 \pm 11.19$ & $52.06 \pm 11.76$ & 0.86 \\
\hline \multicolumn{2}{|c|}{ Education (years) (mean \pm SD) } & $11.75 \pm 4.78$ & $12.19 \pm 4.83$ & 0.73 \\
\hline \multirow[t]{2}{*}{ Sex } & Male & $7 / 16$ & $7 / 16$ & 1.00 \\
\hline & Female & $9 / 16$ & $9 / 16$ & \\
\hline \multirow[t]{2}{*}{ Onset of disease } & Bulbar & $3 / 16$ & & \\
\hline & Extremities & $13 / 16$ & & \\
\hline \multirow[t]{4}{*}{ Diagnostic level } & Definite & $3 / 16$ & & \\
\hline & Probable & $5 / 16$ & & \\
\hline & $\begin{array}{l}\text { Lab-supported } \\
\text { probable }\end{array}$ & $7 / 16$ & & \\
\hline & Possible & $1 / 16$ & & \\
\hline \multirow[t]{2}{*}{ Disease stage } & Advanced ALS & $8 / 16$ & & \\
\hline & Early ALS & $8 / 16$ & & \\
\hline \multicolumn{2}{|c|}{ Duration of illness (months) (mean \pm SD) } & $14.69 \pm 9.16$ & & \\
\hline \multicolumn{2}{|l|}{ Bulbar involvement } & $12 / 16$ & & \\
\hline \multicolumn{2}{|c|}{ ALSFRS-R (mean \pm SD) } & $36.75 \pm 7.42$ & & \\
\hline \multicolumn{2}{|c|}{ Life span (months) (mean \pm SD) } & $48.25 \pm 14.99$ & & \\
\hline \multicolumn{2}{|l|}{ Gene mutation } & $0 / 16$ & & \\
\hline
\end{tabular}

ALSFRS-R, revised ALS functional rating scale; MMSE, mini-mental state examination. Gene mutation includes C9orf72, SOD1, FUS, and TDP43.

quantification in a fully automatic mode. Given the T1weighted MRI data, several brain structures and three major brain tissues are segmented automatically based on prior anatomical knowledge specified by experienced radiologists. The anatomical information is automatically transformed onto the individual brain. The absolute volume (in $\mathrm{ml}$ ) of 20 brain structures and their bilateral components were automatically calculated. These measures and structures included but were not limited to intracranial volume, whole-brain parenchyma, hippocampus, amygdala, ventricular system, lateral ventricle, third ventricle, inferior lateral ventricle, caudate, putamen, pallidum, hypothalamus, thalamus, pons, midbrain, and gray matter in different lobes.

\section{Statistics}

During enrollment, although trying to meet the similarity between the ALS and control groups in sex, age, and education, paired enrollment was still not strictly performed. Therefore, when comparing the clinical characteristics between the ALS and control groups, the chi-square test was used for sex. For age and education factors, $t$-tests were used when the variables were normally distributed, and Mann-Whitney $U$ tests were used when the variables were not normally distributed. Multivariate analysis of covariance (MANCOVA) was used to compare the differences in thalamic and hypothalamic volumes between patients with ALS and controls. During the univariate analysis, when studying the relationship between brain volumes and age, years of education, duration of illness, revised functional rating scale (FRS-R) scores and life span, Pearson correlations were used when the data were continuous, normally distributed and had a linear relationship; otherwise, Spearman correlations were performed. When studying the relationship between brain volumes and sex, bulbar involvement and disease stage, $t$-tests were used when the variables were normally distributed, and Mann-Whitney $U$ tests were used when the variables were not normally distributed. ANOVA was used to compare the brain volumes among different diagnostic levels. Then, multivariable linear regression analysis was used. The standard statistical significance level was set at $p<0.05$. IBM SPSS V20 software was used for all statistical analyses.

\section{RESULTS}

\section{Comparisons of Thalamic Volume Between Patients With Amyotrophic Lateral Sclerosis and Healthy Controls}

Multivariate analysis of covariance was used, in which volumes in the left thalamus and right thalamus were set as two dependent variables; patients with ALS or healthy controls were set as independent variables; and whole-brain volume, age, sex, and education years were set as covariates. The volumes were normally distributed. The patients with ALS and controls were two independent groups. There was homogeneity of variancecovariance matrices assessed using Box's $M$ test of equality of covariance $(F=1.249, p=0.290)$. However, there was no significant difference between the ALS and control groups $(F=1.311, p=0.287)$. Descriptive measures of brain volumes were listed in Supplementary Table 1.

\section{Comparisons of Hypothalamic Volume Between Patients With Amyotrophic Lateral Sclerosis and Healthy Controls}

Multivariate analysis of covariance was used, in which volumes in the left hypothalamus and right hypothalamus were set as two dependent variables; The patients with ALS or healthy controls were set as independent variables; and whole-brain volume, age, sex, and education years were set as covariates. The volumes were normally distributed. The patients with ALS and controls were two independent groups. There was homogeneity of variance-covariance matrices assessed using Box's $M$ test of equality of covariance $(F=2.533, p=0.055)$. However, there was no significant difference between the ALS and control groups $(F=1.647, p=0.213)$. Descriptive measures of brain volumes are listed in Supplementary Table 1.

\section{Thalamic and Hypothalamic Volumes and Clinical Characteristics}

Univariate analysis was first used to choose possible variants for multivariable linear regression analysis. Variants with $p<0.1$ were chosen (Table 2). Descriptive measures of categorical variables, such as disease stages, sex, and bulbar involvement, are listed in Supplementary Table 2.

Multivariable linear regression analysis was separately performed with bilateral thalamic and hypothalamic volumes as the dependent variables and whole-brain volume and factors chosen above as the independent variables. The results are shown in Tables 3, 4. However, after Bonferroni correction $(p<0.0125)$, left and right hypothalamic volumes were 
TABLE 2 | Univariate analysis of brain volumes and clinical characteristics.

\begin{tabular}{|c|c|c|c|c|}
\hline & Left thalamus & Right thalamus & Left hypothalamus & Right hypothalamus \\
\hline Age $(r, p)$ & $-0.54,0.03$ & $-0.61,0.01$ & $0.17,0.54$ & $0.21,0.44$ \\
\hline $\operatorname{Sex}(t, p)$ & $0.21,0.84$ & $0.17,0.87$ & $1.92,0.08$ & $1.93,0.08$ \\
\hline Years of education (r, p) & $0.45,0.08$ & $0.41,0.12$ & $0.27,0.31$ & $0.37,0.15$ \\
\hline Duration of illness (r, p) & $-0.07,0.79$ & $-0.08,0.76$ & $-0.20,0.46$ & $-0.23,0.39$ \\
\hline Bulbar involved (t, p) & $0.59,0.57$ & $0.68,0.51$ & $0.80,0.44$ & $0.38,0.71$ \\
\hline Diagnostic level (F, p) & $2.54,0.11$ & $2.07,0.16$ & $2.30,0.13$ & $2.46,0.11$ \\
\hline Disease stage (t, p) & $0.35,0.73$ & $0.01,0.99$ & $2.16,0.05$ & $2.22,0.04$ \\
\hline ALSFRS-R score $(r, p)$ & $-0.07,0.79$ & $-0.13,0.64$ & $0.28,0.29$ & $0.07,0.79$ \\
\hline Life span (r, p) & $0.12,0.78$ & $0.02,0.95$ & $0.25,0.55$ & $-0.17,0.69$ \\
\hline
\end{tabular}

Factors with $p<0.1$ were chosen as variants for multiple linear regression.

TABLE 3 | Multiple linear regression of brain volumes and clinical characteristics in ALS.

\begin{tabular}{|c|c|c|c|c|}
\hline & Left thalamus & Right thalamus & Left hypothalamus & Right hypothalamus \\
\hline Regression model $\left(\mathrm{R}^{2}\right)$ & 0.63 & 0.56 & 0.70 & 0.66 \\
\hline Regression model (F, p) & $6.70,0.007^{\star}$ & $5.11,0.017^{\star}$ & $6.34,0.007^{\star}$ & $5.30,0.013^{\star}$ \\
\hline Age $(t, p)$ & $2.76,0.017^{\star}$ & $2.83,0.015^{\star}$ & $1.70,0.117$ & $0.93,0.374$ \\
\hline Whole-brain volume (t, p) & $2.21,0.047^{\star}$ & $1.64,0.127$ & $3.19,0.009^{*}$ & $3.03,0.011^{*}$ \\
\hline Education year (t, p) & $1.12,0.283$ & $0.69,0.502$ & - & - \\
\hline $\operatorname{Sex}(t, p)$ & - & - & $0.35,0.731$ & $0.55,0.591$ \\
\hline Disease stage (t, p) & - & - & $0.24,0.813$ & $0.21,0.835$ \\
\hline
\end{tabular}

Four multiple linear regression models were separately made for patients with $A L S,{ }^{*} p<0.05$.

TABLE 4 | Multiple linear regression of brain volumes and clinical characteristics in controls.

\begin{tabular}{|c|c|c|c|c|}
\hline & Left thalamus & Right thalamus & Left hypothalamus & Right hypothalamus \\
\hline Regression model $\left(\mathrm{R}^{2}\right)$ & 0.70 & 0.73 & 0.73 & 0.48 \\
\hline Regression model $(F, p)$ & $9.42,0.002^{*}$ & $10.91,0.001^{*}$ & $10.60,0.001^{*}$ & $3.65,0.045^{\star}$ \\
\hline Age $(t, p)$ & $2.44,0.031^{\star}$ & $2.65,0.021^{*}$ & $1.05,0.316$ & $0.67,0.519$ \\
\hline Whole-brain volume (t, p) & $4.26,0.001^{\star}$ & $4.52,0.001^{\star}$ & $2.31,0.040^{\star}$ & $1.93,0.078$ \\
\hline Education year (t, p) & $0.59,0.569$ & $0.76,0.460$ & - & - \\
\hline $\operatorname{Sex}(t, p)$ & - & - & $3.00,0.011^{*}$ & $1.17,0.265$ \\
\hline
\end{tabular}

Four multiple linear regression models were separately made for controls, * $p<0.05$.

correlated with whole-brain volume in the patients with ALS, while the correlation between age and bilateral thalamic volumes tended to be significant. In the control group, left and right thalamic volumes were correlated with whole-brain volume, and sex was correlated with left hypothalamus volume.

\section{Validation of the Automatic Segmentation of the Thalamus}

Validation of the automatic segmentation of the thalamus was conducted by comparing the automatically generated

TABLE 5 | Validation of the automatic segmentations of thalamus and hypothalamus.

\begin{tabular}{lcc}
\hline Brain region & Mean dice & Min-max \\
\hline Left thalamus & 0.998 & $0.990-1.000$ \\
Right thalamus & 0.999 & $0.986-1.000$ \\
Left hypothalamus & 0.995 & $0.958-1.000$ \\
Right hypothalamus & 0.994 & $0.967-1.000$
\end{tabular}

segmentation labels with the corrected labels manually performed based on the automatic results. The dice similarity coefficient (range: $0-1$ ) was used as the metric of segmentation accuracy (Table 5).

\section{DISCUSSION}

In this study, hypothalamic volume was not different $(F=1.65$, $p=0.213$ ) between the patients with ALS and healthy controls after adjusting for age, sex, education year, and wholebrain volume. Although relatively few studies have reported hypothalamic volume in patients with ALS, Cykowski and Nakamura found pathological changes in ALS autopsy (Cykowski et al., 2014; Nakamura et al., 2015), and Gorges found hypothalamic atrophy in patients with ALS (Gorges et al., 2017). The probable reasons for the negative result in this study include the following: (1) the study did not include subregional analysis of the hypothalamus, which might have covered some potential significance; (2) the sensitivity of AccuBrain ${ }^{\circledR}$ software: Although 
the study provided a comparison with post-segmentation manual correction, it was not comparable to validation studies of alternative software (Wolff et al., 2018; Billot et al., 2020); and (3) the sample of this study was small, which might have affected the accuracy of the analyses.

Thalamic volume was also not different $(F=1.31, p=0.287)$ between the patients with ALS and healthy controls after adjusting for age, sex, education year, and whole-brain volume in this study. There were actually more reports of thalamic atrophy than hypothalamic atrophy in the patients with ALS (Douaud et al., 2011; Sharma et al., 2011, 2013; Machts et al., 2015; Westeneng et al., 2016; Christidi et al., 2018; Chipika et al., 2020), although this study did not show a difference. In addition to the probable reasons listed as hypothalamic volume, thalamic atrophy was most commonly reported in the patients with ALS who also had frontotemporal dysfunctions (Machts et al., 2015; Westeneng et al., 2016; Christidi et al., 2018). Other studies did not mention cognitive function (Douaud et al., 2011; Sharma et al., 2011, 2013). Patients with normal cognitive function have seldom been reported. Since the cognition of this group of the patients with ALS was normal, we hypothesize that thalamic atrophy in the patients with ALS may be more related to frontotemporal dysfunction and needs further study.

Age is commonly recognized to have a negative correlation with brain volumes. However, although there were trends of significance between age and thalamic volume, there were no significant differences between age and hypothalamic volume in either the patients with ALS or controls. Gorges's (Gorges et al., 2017) study found that the age at the onset was correlated with anterior hypothalamic volume but not with total or posterior hypothalamic volumes. Our study did not divide the hypothalamus into anterior and posterior parts; thus, it is unknown whether any subregional differences exist. It is also worth noting that, unlike the thalamus, hypothalamic volume was not correlated with whole-brain volume in healthy controls but was significantly correlated in the patients with ALS. Thus, we speculate that hypothalamic volume may be a potential age-independent biomarker for ALS. Additionally, hypothalamic volume was not correlated with the disease stage. These characteristics suggest that further in-depth investigation is required to determine the structural and functional roles of the hypothalamus.

Generally, there are some limitations of this study. First, the sample size was small, which may have affected the accuracy of the analyses, and the results need confirmation in further large-sample studies. Second, we did not divide subregions of the thalamus and hypothalamus, which may have different brain network connections. Third, we used the AccuBrain ${ }^{\circledR}$ technique to analyze thalamic and hypothalamic volumes. The software is commercial and inaccessible to other researchers; therefore, it is not easy to replicate by fellow colleagues. As our study did not provide commonly accepted measures but only a comparison with the post-segmentation manual correction, it is not comparable to validation studies assessing alternative software. For future work, it will be important to compare volumes by using these different sensitive techniques to confirm the results. Much work remains to be done for automated thalamus and hypothalamus volumetry, requiring larger sample sizes to compensate for the measurement errors and to provide a deeper understanding of the role of the thalamus and hypothalamus.

\section{CONCLUSION}

Thalamic and hypothalamic volumes did not show differences between the patients with normal frontotemporal function ALS and healthy controls, but further studies are needed.

\section{DATA AVAILABILITY STATEMENT}

The original contributions presented in the study are included in the article/Supplementary Material, further inquiries can be directed to the corresponding author/s.

\section{ETHICS STATEMENT}

The studies involving human participants were reviewed and approved by the Ethics Committee of PUTH. The patients/participants provided their written informed consent to participate in this study. Written informed consent was obtained from the individual(s) for the publication of any potentially identifiable images or data included in this article.

\section{AUTHOR CONTRIBUTIONS}

DF conceived this study and provided financial support. SY, LS, and DF designed the research. SY and LS analyzed the data and wrote the manuscript. PJ, YW, NZ, GZ, and LC collected the data. YL analyzed the data. DF was responsible for project management. All authors contributed to the article and approved the submitted version.

\section{FUNDING}

This work was supported by grants from the National Natural Science Foundation of China (82001350 and 81873784), PUTH Key Clinical Projects (2018048) and Cohort Construction Project (DL2019002), and the Research Grants Council of the Hong Kong Special Administrative Region, China (CUHK14204117), and Beijing E-Town Cooperation \& Development Foundation (YJXJ-JZ-2021-0014).

\section{SUPPLEMENTARY MATERIAL}

The Supplementary Material for this article can be found online at: https://www.frontiersin.org/articles/10.3389/fnagi.2021. 610332/full\#supplementary-material 


\section{REFERENCES}

Abrigo, J., Shi, L., Luo, Y., Chen, Q., Chu, W. C. W., Mok, V. C. T., et al. (2018). Standardization of hippocampus volumetry using automated brain structure volumetry tool for an initial Alzheimer's disease imaging biomarker. Acta Radiol. 60, 769-776. doi: 10.1177/0284185118795327

Ahmed, R. M., Irish, M., Piguet, O., Halliday, G. M., Ittner, L. M., Farooqi, S., et al. (2016). Amyotrophic lateral sclerosis and frontotemporal dementia: distinct and overlapping changes in eating behaviour and metabolism. Lancet Neurol. 15, 332-342. doi: 10.1016/S1474-4422(15)00380-4

Ahmed, R. M., Landin-Romero, R., Collet, T. H., Van der Klaauw, A. A., Devenney, E., Henning, E., et al. (2017). Energy expenditure in frontotemporal dementia: a behavioural and imaging study. Brain 140, 171-183. doi: 10.1093/brain/ aww263

Balendra, R., and Isaacs, A. M. (2018). C9orf72-mediated ALS and FTD: multiple pathways to disease. Nat. Rev. Neurol. 14, 544-558. doi: 10.1038/s41582-018$0047-2$

Billot, B., Bocchetta, M., Todd, E., Dalca, A. V., Rohrer, J. D., and Iglesias, J. E. (2020). Automated segmentation of the hypothalamus and associated subunits in brain MRI. Neuroimage 223:117287. doi: 10.1016/j.neuroimage.2020.11 7287

Bocchetta, M., Gordon, E., Manning, E., Barnes, J., Cash, D. M., Espak, M., et al. (2015). Detailed volumetric analysis of the hypothalamus in behavioral variant frontotemporal dementia. J. Neurol. 262, 2635-2642.

Brook, B. R., Miller, R. G., Swash, M., and Munsat, T. L. World federation of neurology research group on motor neuron diseases. El Escorial revisited: revised criteria for the diagnosis of amyotrophic lateral sclerosis. Amyotroph. Lateral Scler. Other Motor Neuron Disord. 1, 293-299. doi: 10.1080/ 146608200300079536

Chipika, R. H., Finegan, E., Shing, L. H., McKenna, M. C., Christidi, F., Chang, K. M., et al. (2020). "Switchboard" malfunction in motor neuron diseases: selective pathology of thalamic nuclei in amyotrophic lateral sclerosis and primary lateral sclerosis. Neuroimage Clin. 27:102300. doi: 10.1016/j.nicl.2020. 102300

Christidi, F., Karavasilis, E., Riederer, F., Zalonis, I., Ferentinos, P., Velonakis, G., et al. (2018). Gray matter and white matter changes in non-demented amyotrophic lateral sclerosis patients with or without cognitive impairment: a combined voxel-based morphometry and tract-based spatial statistics wholebrain analysis. Brain Imaging Behav. 12, 547-563.

Congiu, P., Mariani, S., Milioli, G., Parrino, L., Tamburrino, L., Borghero, G., et al. (2019). Sleep cardiac dysautonomia and EEG oscillations in amyotrophic lateral sclerosis. Sleep 42:zsz163. doi: 10.1093/sleep/zsz164

Cykowski, M. D., Takei, H., Schulz, P. E., Appel, S. H., and Powell, S. Z. (2014). TDP-43 pathology in the basal forebrain and hypothalamus of patients with amyotrophic lateral sclerosis. Acta Neuropathol. Commun. 2:171. doi: 10.1186/ s40478-014-0171-1

Diaz-Abad, M., Buczyner, J. R., Venza, B. R., Scharf, S. M., Kwan, J. Y., Lubinski, B., et al. (2018). Poor sleep quality in patients with amyotrophic lateral sclerosis at the time of diagnosis. J. Clin. Neuromuscul. Dis. 20, 60-68. doi: 10.1097/CND. 0000000000000234

Dorst, J., Dupuis, L., Petri, S., Kollewe, K., Abdulla, S., Wolf, J., et al. (2015). Percutaneous endoscopic gastrostomy in amyotrophic lateral sclerosis: a prospective observational study. J. Neurol. 262, 849-858. doi: 10.1007/s00415015-7646-2

Dou, W., Zhao, L., Su, C., Lu, Q., Liu, Q., Guo, J., et al. (2020). A quantitative MRI index for assessing the severity of hippocampal sclerosis in temporal lobe epilepsy. BMC Med. Imaging 20:42. doi: 10.1186/s12880-020-00440-z

Douaud, G., Filippini, N., Knight, S., Talbot, K., and Turner, M. R. (2011). Integration of structural and functional magnetic resonance imaging in amyotrophic lateral sclerosis. Brain 134, 3470-3479. doi: 10.1093/brain/awr279

Dupuis, L., Pradat, P. F., Ludolph, A. C., and Loeffler, J. P. (2011). Energy metabolism in amyotrophic lateral sclerosis. Lancet Neurol. 10, 75-82. doi: 10.1016/S1474-4422(10)70224-70226

Foerster, B. R., Welsh, R. C., and Feldman, E. L. (2013). 25 years of neuroimaging in amyotrophic lateral sclerosis. Nat. Rev. Neurol. 9, 513-524. doi: 10.1038/ nrneurol.2013.153

Gallo, V., Wark, P. A., Jenab, M., Pearce, N., Brayne, C., Vermeulen, R., et al. (2013). Prediagnostic body fat and risk of death from amyotrophic lateral sclerosis: the EPIC cohort. Neurology 80, 829-838. doi: 10.1212/WNL.0b013e3182840689
Gijselinck, I., Langenhove, T. V., Zee, J., Sleegers, K., Philtjens, S., Kleinberger, G., et al. (2012). A C9orf72 promoter repeat expansion in a Flanders-Belgian cohort with disorders of the frontotemporal lobar degeneration-amyotrophic lateral sclerosis spectrum: a gene identification study. Lancet Neurol. 11, 54-65. doi: $10.1016 /$ S1474-4422(11)70261-7

Gorges, M., Vercruysse, P., Müller, H. P., Huppertz, H. J., Rosenbohm, A., Nagel, G., et al. (2017). Hypothalamic atrophy is related to body mass index and age at onset in amyotrophic lateral sclerosis. J. Neurol. Neurosurg. Psychiatry 88, 1033-1041. doi: 10.1136/jnnp-2017-315795

Guo, C., Niu, K., Luo, Y., Shi, L., Wang, Z., Zhao, M., et al. (2019). Intra-scanner and inter-scanner reproducibility of automatic white matter hyperintensities quantification. Front. Neurosci. 13:679. doi: 10.3389/fnins.2019.00679

Hou, B., Gao, L., Shi, L., Luo, Y., Guo, X., Young, G., et al. (2020). Reversibility of impaired brain structures after transsphenoidal surgery in Cushing's disease: a longitudinal study based on an artificial intelligence-assisted tool. J. Neurosurg. 1, 1-10. doi: 10.3171/2019.10.JNS191400

Huisman, M. H., Seelen, M., van Doormaal, P. T., de Jong, S. W., de Vries, J. H., van der Kooi, A. J., et al. (2015). Effect of presymptomatic body mass index and consumption of fat and alcohol on amyotrophic lateral sclerosis. JAMA Neurol. 72, 1155-1162. doi: 10.1001/jamaneurol.2015.1584

Liu, C., Shi, L., Zhu, W., Yang, S., Sun, P., Qin, Y., et al. (2020). Fiber connectivity density in cerebral small-vessel disease patients with mild cognitive impairment and cerebral small-vessel disease patients with normal cognition. Front. Neurosci. 14:83. doi: 10.3389/fnins.2020.00083

Liu, R., Sheng, Z., Cai, B., Zhang, Y., and Fan, D. (2015). Increased orexin expression promotes sleep/wake disturbances in the SOD1-G93A mouse model of amyotrophic lateral sclerosis. Chin. Med. J (Engl) 128, 239-244. doi: 10.4103/ 0366-6999.149214

Liu, S., Huang, Y., Tai, H., Zhang, K., Wang, Z., Shen, D., et al. (2018). Excessive daytime sleepiness in Chinese patients with sporadic amyotrophic lateral sclerosis and its association with cognitive and behavioural impairments. J. Neurol. Neurosurg. Psychiatry 89, 1038-1043. doi: 10.1136/jnnp-2018-318810

Machts, J., Loewe, K., Kaufmann, J., Jakubiczka, S., Abdulla, S., Petri, S., et al. (2015). Basal ganglia pathology in ALS is associated with neuropsychological deficits. Neurology 85, 1301-1309.

Nakamura, M., Bieniek, K. F., Lin, W. L., Graff-Radford, N. R., Murray, M. E. Castanedes-Casey, M., et al. (2015). A truncating SOD1 mutation, p.gly141x, is associated with clinical and pathologic heterogeneity, including frontotemporal lobar degeneration. Acta Neuropathol. 130, 145-157. doi: 10.1007/s00401-0151431-2

O’Reilly, E. J., Wang, H., Weisskopf, M. G., Fitzgerald, K. C., Falcone, G., McCullough, M. L., et al. (2013). Premorbid body mass index and risk of amyotrophic lateral sclerosis. Amyotroph. Lateral Scler. Frontotemporal Degener. 14, 205-211. doi: 10.3109/21678421.2012.735240

Schönecker, S., Neuhofer, C., Otto, M., Ludolph, A., Kassubek, J., Landwehrmeyer, B., et al. (2018). Atrophy in the thalamus but not cerebellum is specific for C9orf72 FTD and ALS patients - an atlas-based volumetric MRI study. Front Aging Neurosci. 10:45. doi: 10.3389/fnagi.2018.00045

Sharma, K. R., Saigal, G., Maudsley, A. A., and Govind, V. (2011). 1H MRS of basal ganglia and thalamus in amyotrophic lateral sclerosis. NMR Biomed. 24, $1270-1276$

Sharma, K. R., Sheriff, S., Maudsley, A., and Govind, V. (2013). Diffusion tensor imaging of basal ganglia and thalamus in amyotrophic lateral sclerosis. J. Neuroimaging 23, 368-374.

Strong, M. J., Abrahams, S., Goldstein, L. H., Woolley, S., Mclaughlin, P., Snowden, J., et al. (2017). Amyotrophic lateral sclerosis - frontotemporal spectrum disorder (ALS-FTSD): revised diagnostic criteria. Amyotroph. Lateral Scler. Frontotemporal. Degener. 18, 153-174. doi: 10.1080/21678421.2016.1267768

van Es, M. A., Hardiman, O., Chio, A., Al-Chalabi, A., Pasterkamp, R. J., Veldink, J. H., et al. (2017). Amyotrophic lateral sclerosis. Lancet 390, 2084-2098. doi: 10.1016/S0140-6736(17)31287-4

Vercruysse, P., Sinniger, J., Oussini, H. E., Scekic-Zahirovic, J., Dieterlé, S., Dengler, R., et al. (2016). Alterations in the hypothalamic melanocortin pathway in amyotrophic lateral sclerosis. Brain 139(Pt 4), 1106-1122. doi: 10.1093/brain/ aww004

Vercruysse, P., Vieau, D., Blum, D., Petersén, A., and Dupuis, L. (2018). Hypothalamic alterations in neurodegenerative diseases and their relation to abnormal energy metabolism. Front. Mol. Neurosci. 11:2. doi: 10.3389/fnmol. 2018.00002 
Wang, C., Zhao, L., Luo, Y., Liu, J., Miao, P., Wei, S., et al. (2019). Structural covariance in subcortical stroke patients measured by automated MRIbased volumetry. NeuroImage Clin. 22:101682. doi: 10.1016/j.nicl.2019.10 1682

Westeneng, H. J., Walhout, R., Straathof, M., Schmidt, R., Hendrikse, J., Veldink, J. H., et al. (2016). Widespread structural brain involvement in ALS is not limited to the C9orf72 repeat expansion. J. Neurol. Neurosurg. Psychiatry 87, 1354-1360. doi: 10.1136/jnnp-2016-313959

Wills, A. M., Hubbard, J., Macklin, E. A., Glass, J., Tandan, R., Simpson, E. P., et al. (2014). Hypercaloric enteral nutrition in patients with amyotrophic lateral sclerosis: a randomized, double-blind, placebo-controlled phase 2 trial. Lancet 383, 2065-2072. doi: 10.1016/S0140-6736(14)60 222-1

Wolff, J., Schindler, S., Lucas, C., Binninger, A., Weinrich, L., Schreiber, J., et al. (2018). A semi-automated algorithm for hypothalamus volumetry in 3 Tesla magnetic resonance images. Psychiatry Res. Neuroimaging 277, 45-51. doi: 10. 1016/j.pscychresns.2018.04.007

Zhang, T., Jiang, X., Xu, M., Wang, H., Sang, X., Qin, M., et al. (2018). Sleep and circadian abnormalities precede cognitive deficits in R521C FUS knockin rats. Neurobiol. Aging 72, 159-170. doi: 10.1016/j.neurobiolaging.2018. 08.025

Zhao, L., Luo, Y., Lew, D., Liu, W., Au, L., Mok, V., et al. (2019). Risk estimation before progression to mild cognitive impairment and Alzheimer's disease: an
$\mathrm{AD}$ resemblance atrophy index. Aging 11, 6217-6236. doi: 10.18632/aging 102184

Conflict of Interest: LS is the director of BrainNow Medical Technology Limited. YL is now employed by BrainNow Medical Technology Limited.

The remaining authors declare that the research was conducted in the absence of any commercial or financial relationships that could be construed as a potential conflict of interest.

Publisher's Note: All claims expressed in this article are solely those of the authors and do not necessarily represent those of their affiliated organizations, or those of the publisher, the editors and the reviewers. Any product that may be evaluated in this article, or claim that may be made by its manufacturer, is not guaranteed or endorsed by the publisher.

Copyright (c) 2022 Ye, Luo, Jin, Wang, Zhang, Zhang, Chen, Shi and Fan. This is an open-access article distributed under the terms of the Creative Commons Attribution License (CC BY). The use, distribution or reproduction in other forums is permitted, provided the original author(s) and the copyright owner(s) are credited and that the original publication in this journal is cited, in accordance with accepted academic practice. No use, distribution or reproduction is permitted which does not comply with these terms. 